\title{
Silencing of TGM2 reverses epithelial to mesenchymal transition and modulates the chemosensitivity of breast cancer to docetaxel
}

\author{
WENXING HE ${ }^{1}$, ZHENGKUI SUN $^{2}$ and ZHIMING LIU ${ }^{1}$ \\ ${ }^{1}$ Department of General Surgery, The First Affiliated Hospital of Guangxi Medical University, Nanning, Guangxi 530021; \\ ${ }^{2}$ Department of Breast Surgery, Jiangxi Provincial Cancer Hospital, Nanchang, Jiangxi 330029, P.R. China
}

Received September 21, 2014; Accepted July 9, 2015

DOI: $10.3892 / \mathrm{etm} .2015 .2679$

\begin{abstract}
Epithelial to mesenchymal transition (EMT) plays a critical role in drug resistance. The aim of the present study was to further elucidate its role by examining the effect of tissue transglutaminase (TG2) on EMT and drug resistance in breast cancer. An antisense lentiviral (LV) short hairpin (sh)RNA construct specific to the TG2 gene (TGM2) was designed, synthesized and stably transfected into MDA-MB-231 cells to silence TGM2 by RNA interference (RNAi). The transfected cells expressed low levels of TG2 and constituted the RNAi (TGM2-shRNA) group. A control (NC) group was also established by transfecting MDA-MB-231 cells with scrambled shRNA. The expression levels of TG2, E-cadherin, vimentin and B-cell lymphoma (Bcl)-2 in the cells were examined via western blotting. The transfected MDA-MB-231 cells were divided into four groups, two of which were treated with doxetaxel (TXT): NC, RNAi, TXT and RNAi + TXT groups, Cell proliferation was analyzed by MTT assay and cell apoptosis was detected by flow cytometry. An in vivo assay was also conducted, in which MDA-MB-231 cells transfected with scrambled shRNA or TGM2-shRNA were subcutaneously implanted into nude mice. After 2 weeks, TXT or vehicle was intraperitoneally administered at a dose of $10 \mathrm{mg} / \mathrm{kg}$ on day 1 of every week and tumor growth was monitored. Following the silencing of TGM2 in the MDA-MB-231 cells, the cells showed changes in morphology, indicating that an increased expression of TG2 was associated with a mesenchymal morphology. Transfection of the cells with TGM2-shRNA affected the expression of TG2, E-cadherin, vimentin and $\mathrm{Bcl}-2$. In the MTT assay, the proliferation of MDA-MB-231 cells was significantly inhibited in the RNAi group compared with the control group $(\mathrm{P}<0.05)$, and the inhibitory effect
\end{abstract}

Correspondence to: Professor Zhiming Liu, Department of General Surgery, The First Affiliated Hospital of Guangxi Medical University, 6 Shuangyong Road, Nanning, Guangxi 530021, P.R. China

E-mail:1zmhwx@126.com

Key words: docetaxel, transglutaminase 2, breast cancer, epithelial to mesenchymal transition, drug resistance increased in a time-dependent manner. Following treatment with TXT for $48 \mathrm{~h}$, apoptosis was significantly promoted in the RNAi + TXT group compared with that in the other groups $(\mathrm{P}<0.05)$. Measurement of the tumors in the nude mice indicated that the combination of RNAi and TXT brought about a stronger antitumor effect than either treatment alone. These results suggest that the downregulation of TG2 reversed EMT and modulated the chemosensitivity of breast cancer to TXT. TG2 may be an important predictive and prognostic factor for the treatment efficacy of chemotherapy in patients with breast cancer.

\section{Introduction}

Triple-negative breast cancer (TNBC), which comprises $15-20 \%$ of all breast cancers, has a low survival rate due to drug resistance. It is imperative for new therapeutic targets to be identified in order to improve the outlook for these patients. Epithelial to mesenchymal transition (EMT) plays a critical role in drug resistance (1). EMT is a process by which epithelial cells lose their cell polarity and cell-cell adhesion, and gain migratory and invasive properties. The process also controls chemoresistance and immune escape (2).

Tissue transglutaminase (TG2) belongs to the family of transglutaminase enzymes that are active in the presence of $\mathrm{Ca}^{2+}$, and catalyze $\mathrm{Ca}^{2+}$-dependent protein crosslinking via the formation of amide bonds (3). It has been reported that TG2 induces EMT in MCF10A and MCF12A mammary epithelial cells (4). Docetaxel (TXT) is one of the most effective chemotherapy drugs and TXT chemotherapy is widely used to treat breast cancer.

When the clinical and biological significance of TG2 was examined in ovarian cancer (5), knockdown of the TG2 gene (TGM2) of HeyA8 cells with small interfering (si)RNA strongly promoted TXT-induced cell death. Mouse model studies indicate that TG2 is a potential therapeutic target for chemo-resistant ovarian cancer (5). The combination of TGM2-siRNA and TXT with chitosan hydrogel facilitated a greater inhibition of cancer growth compared with that achieved using TXT and chitosan hydrogel (92 vs. 55\% reduction; $\mathrm{P}<0.001)(6)$.

Drug resistance poses a major challenge to the treatment of breast cancer and until now little has been known about the role that TG2 plays in breast cancer. It may be speculated that 
TG2 is involved in EMT and TXT-induced cell death in breast cancer. To better understand the effect of TG2 on drug resistance, the role of TG2 in EMT and drug resistance in breast cancer was examined in the present study. Breast cancer cells were modified by TGM2-specific RNA interference (RNA)i and the effect of TG2 on the sensitivity of breast cancer to TXT was investigated in vitro and in xenograft tumor models in nude mice.

\section{Materials and methods}

Cell line and materials. MDA-MB-231 TNBC cells were purchased from the Shanghai Institute of Biochemistry and Cell Biology, Chinese Academy of Sciences (Shanghai, China) and cultured in L-15 medium (WISENT, Inc., Nanjing, China) supplemented with $10 \%$ fetal bovine serum (FBS) and $1 \%$ penicillin-streptomycin $(10,000 \mathrm{U} / \mathrm{ml}$ penicillin and $10 \mathrm{mg} / \mathrm{ml}$ streptomycin) at $37^{\circ} \mathrm{C}$ in a humidified atmosphere $\left(\mathrm{CO}_{2}\right.$ was not present).

An antisense lentiviral (LV) RNAi vector targeting the TGM2 gene with short hairpin (sh)RNA (TGM2-shRNA-LV) was designed, synthesized and stably transfected into MDA-MB-231 cells, which subsequently expressed low levels of TG2. The targeting sequence 5'-GCAGTGACT TTGACGTCTT-3' was designed to target the TGM2 gene (GenBank accession No. NM_004613), and was cloned into the lentiviral vector GV115 (Shanghai GeneChem Co. Ltd., Shanghai, China). The specificity was confirmed by a BLAST search of the GenBank database (http://www.ncbi.nlm.nih. gov/genbank/). A green fluorescent protein lentiviral vector containing a non-effective (scrambled) shRNA cassette served as a negative control for gene downregulation.

TXT was purchased from Jiangsu Hengrui Medicine Co. Ltd. (Lianyungang, China). The MDA-MB-231 cells were divided into the RNAi (TGM2-shRNA) and NC (scrambled shRNA) groups and the expression levels of TG2, E-cadherin, vimentin and Bcl-2 in the cells were examined via western blotting.

Western blot analysis. Cultured cells were washed and harvested in a lysis solution containing SDS and NP-40 (Beijing Solarbio Science \& Technology Co., Ltd., Beijing, China). The proteins were separated by SDS-PAGE and transferred to a polyvinylidene difluoride membrane by electroblotting, and then blocked with $2.5 \%$ non-fat milk in Tris-buffered saline with Tween 20 for $2 \mathrm{~h}$ at room temperature. The membranes were then probed with the relevant mouse monoclonal antibodies overnight at $4^{\circ} \mathrm{C}$ : Anti-TG2 (CUB7402), anti-E-cadherin (HECD-1), anti-Bcl-2 (Bcl2/100), anti-GAPDH (9484; Abcam, Cambridge, MA, USA) and anti-vimentin (RV202; Santa Cruz Biotechnology, Inc., La Jolla, CA, USA), which were diluted according to the manufacturer's recommendations. Washing steps were performed using $0.1 \%$ Tris-buffered saline and Tween (5 min x 3). Secondary antibodies were diluted 1:2,000 and incubated for $2 \mathrm{~h}$ at room temperature. Immunoreactive proteins were detected via western blot enhanced chemiluminescence (ECL Plus; Beijing Solarbio Science \& Technology Co., Ltd.). Quantitative densitometry of the electrophoretic bands images was conducted using Image $J$ software (National Institutes of Health, Bethesda, MD, USA). Intensity levels of the target protein were then normalized against those of GAPDH, the house keeping protein.

Detection of cell proliferation by MTT assay. Cells were passed through no. 400 stainless steel meshes and inoculated into 96 -well plates at $1.5 \times 10^{3}$ cells/well. The cells were divided into four groups, two of which were treated with $3.7 \mu \mathrm{g} / \mathrm{ml}$ TXT. These were the NC, RNAi, TXT (NC + TXT) and RNAi + TXT groups. Cells were cultured at $37^{\circ} \mathrm{C}$ for 24 , 48 or $72 \mathrm{~h}$, following which MTT was added. After another $4 \mathrm{~h}$ of culturing, the complete medium containing the drug and the unconverted MTT was removed, $200 \mu \mathrm{l}$ dimethyl sulfoxide was added to each well, and the absorbance of each well was read at $570 \mathrm{~nm}$ using an ELISA microplate reader (ST-360; KHB, Shanghai, China). The growth inhibition rate of the tumor cells was then calculated using the following formula: Inhibition rate $(\%)=[1$-optical density $(\mathrm{OD})$ drug exposure/OD control] x 100. The effective anticancer activity was regarded as sensitive. All experiments were repeated four times under the same conditions.

Detection of cell apoptosis by flow cytometry. The transfected cells were seeded into $25 \mathrm{ml}$ flasks. They were treated with TXT at a concentration of $3.7 \mu \mathrm{g} / \mathrm{ml}$ (the average peak plasma concentration) when they covered $80 \%$ of the flask, or were untreated. Following $48 \mathrm{~h}$ of treatment, the cells were washed with phosphate-buffered saline (PBS), digested with trypsin, pelleted by centrifugation at $300 \mathrm{x} g$ for $10 \mathrm{~min}$, resuspended in an EP tube with PBS, and fixed and permeabilized following the addition of $2 \mathrm{ml}$ ice-cold $70 \%$ ethanol. The cells were washed three times in PBS and Annexin V/propidium iodide (ROTRN Shanghai Biological Technology Co., Ltd., Shanghai, China) was added. The cells were stored at $4^{\circ} \mathrm{C}$, allowed to stand at room temperature in the dark for $3 \mathrm{~min}$, and then filtered through 400-mesh filter traps, prior to analysis of cell apoptosis using a BD FACSCanto II flow cytometer (BD Biosciences, Franklin Lakes, NJ, USA).

Animals and xenograft tumor model study

Animals. The animal experiments were approved by the Ethics Committee of Guangxi Medical University (Nanning, China). Female Balb/c nude mice were purchased from the Medical Laboratory Animal Center of Guangxi Medical University and maintained at the animal laboratory under specific pathogen-free conditions. Six-week old female nude mice, each weighing 20-25 g, were injected with cells into a fat pad of mammary gland. Mice were housed in an animal room, with water and food freely available. The mice $(n=24)$ were randomly divided into four experimental groups $(n=6$ per group).

Xenograft tumor model study. The antitumor effect of combination therapy with TGM2-shRNA and TXT on human breast cancer xenografts derived from MDA-MB-231 cells was investigated. To evaluate the antitumor effects of TGM2-shRNA and TXT in vivo, 5x10 $0^{6}$ MDA-MB-231 cells transfected with scrambled shRNA or TGM2-shRNA vector (in $100 \mu \mathrm{l}$ PBS, pH 7.3) were injected subcutaneously and once daily for 3 days into the right thoracic mammary fat pad of the 6 -week-old female nude mice. Following the injection, the mice were monitored daily for tumor volume and survival. 
A

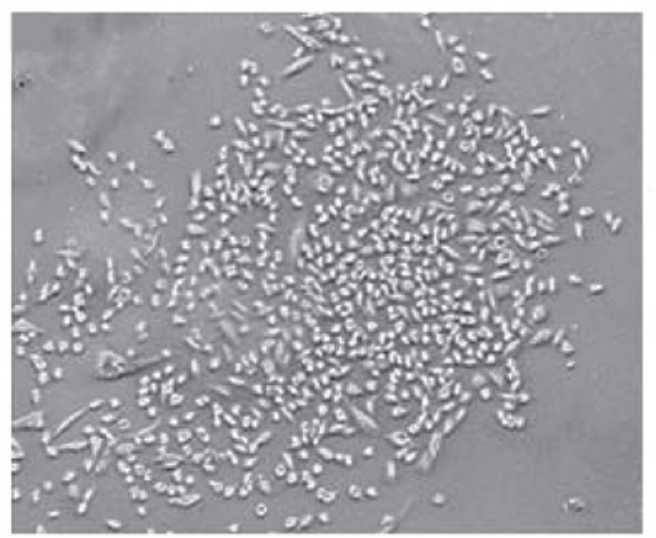

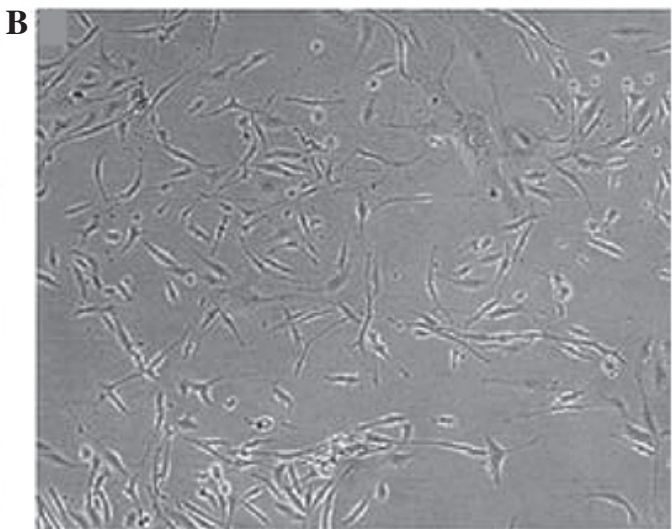

Figure 1. TGM2-shRNA-LV reverses epithelial to mesenchymal transition in MDA-MB-231 cells. MDA-MB-231 cells were stably transfected with (A) TGM2-shRNA-LV construct or (B) scrambled shRNA alone. Images taken following 10 days culture in medium. Magnification, x200. TGM2, transglutaminase 2, shRNA, short hairpin RNA; LV, lentivirus.

After 2 weeks, all 24 animals showed tumor growth, and PBS or TXT $(10 \mathrm{mg} / \mathrm{kg})$ was injected intraperitoneally on day 1 of every week. The tumor volume was measured by vemier caliper every 3 days, and calculated according to the formula: Tumor volume $=\pi / 6 \mathrm{x}$ (width $\mathrm{x}$ length ${ }^{2}$ ), where width and length are the shortest and the longest diameters of the tumor, respectively. The measurements for the MDA-MB-231 xenografts were continued for 3 weeks.

Examination of TG2 expression within tumors. Immunohistochemical analysis was carried out to explore whether TGM2-shRNA effectively downregulated the expression of TG2 in vivo. Xenografted tumors from sacrificed nude mice were collected and fixed in $10 \%$ formalin for immunohistochemical analysis after weighing and measuring.

The analysis of the TG2 expression was conducted using the TG2 mouse monoclonal Ab. The total staining of TG2 was evaluated according to the percentage and intensity of cells with TG2 cytoplasm staining.

Statistical analysis. Data were analyzed with SPSS software (version 17.0; SPSS Inc., Chicago, IL, USA). Student's t-test was used for quantitative results. The $\chi^{2}$ and Fisher's exact tests were used to compare the expression of TG2 within tumors grown in nude mice. Values of $\mathrm{P}<0.05$ were considered to indicate a statistically significant difference.

\section{Results}

Morphological appraisal of breast cancer cells. Following gene silencing of TGM2, the MDA-MB-231 cells showed changes in morphology. The MDA-MB-231 cells in the NC group remained elongated and dispersed whereas the cells in the TGM2-shRNA group were rounded with cobblestone epithelial morphology, and were organized in compact structures (Fig. 1). These data suggest that an increase in the expression of TG2 is associated with a mesenchymal morphology (7).

Expression of TG2, E-cadherin, vimentin and Bcl-2 in $M D A-M B-231$ cells. To analyze the biological consequences of reduced TG2 expression in MDA-MB-231 cells, the expression of endogenous TG2 in MDA-MB-231 cells was inhibited

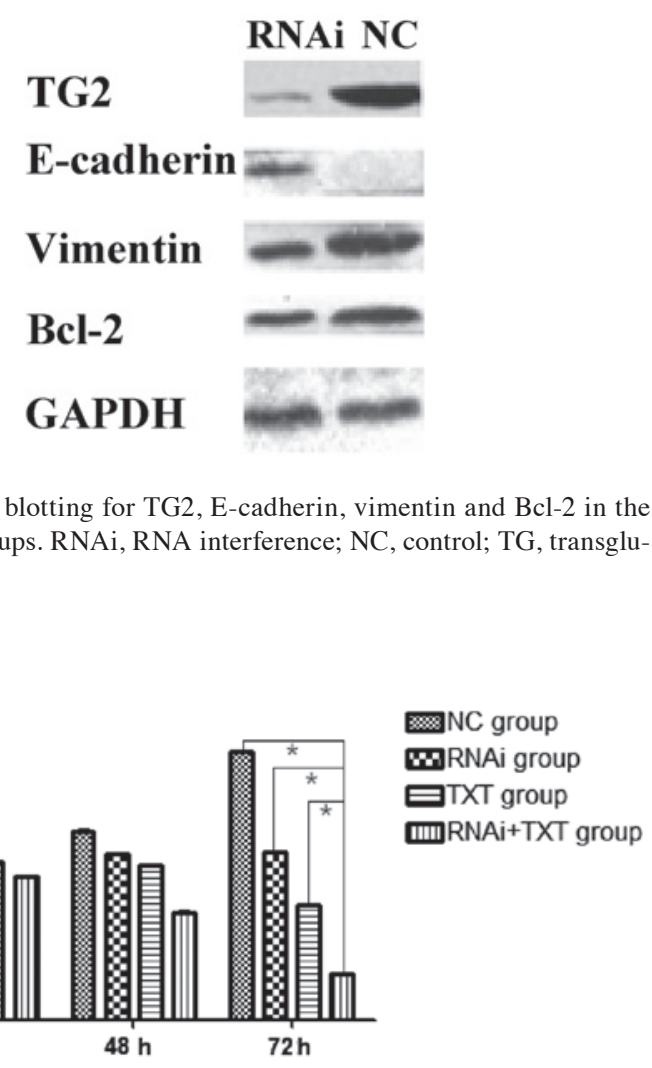

Figure 3. Effect of TXT on the proliferation of MDA-MB-231 cells as evaluated by MTT assay $\left({ }^{*} \mathrm{P}<0.05\right)$. TXT, docetaxel; OD570, optical density at $570 \mathrm{nM}$; RNAi, RNA interference; NC, control.

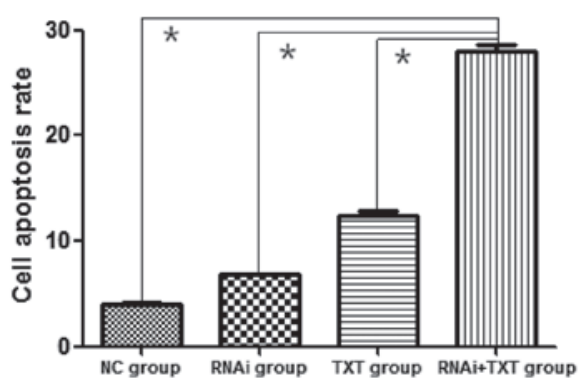

Figure 4. Apoptosis rate of MDA-MB-231 cells ( $\left.{ }^{*} \mathrm{P}<0.05\right)$. TXT, docetaxel; RNAi, RNA interference; NC, control. 

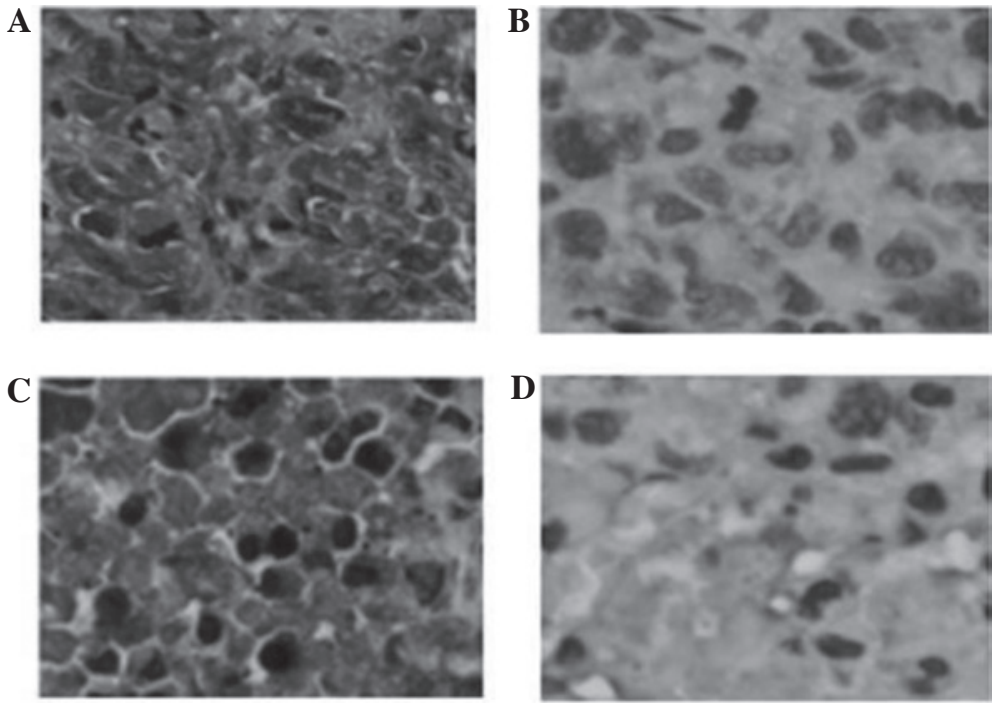

Figure 5. Representative immunohistochemical staining for TG2 in MDA-MB-231 tumors grown in nude mice. (A) NC group; (B) RNAi group; (C) TXT group; (D) RNAi+TXT group. NC and TXT groups show marked expression, while the RNAi and RNAi + TXT group show negative expression. Magnification, x400. TG, transglutaminase; NC, normal control; RNAi, RNA interference; TXT, docetaxel.

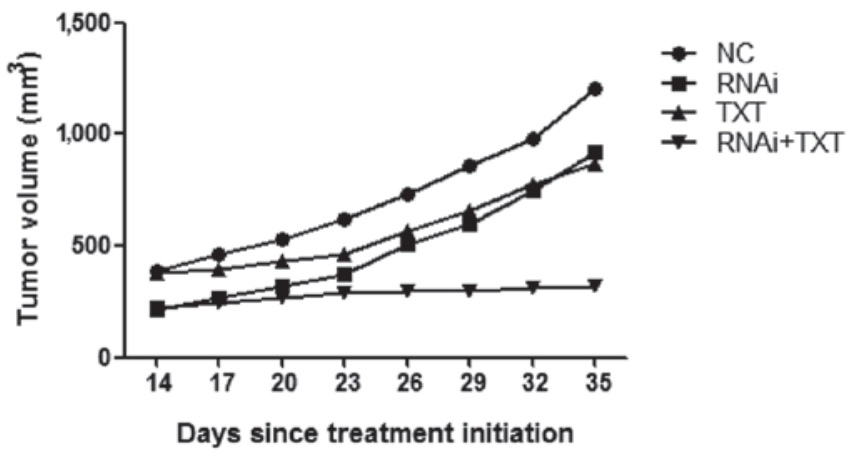

Figure 6. Volume of the tumors in the nude mice. NC, normal control; TXT, docetaxel; RNAi, RNA interference.

by RNAi and the expression of TG2, E-cadherin, vimentin and $\mathrm{Bcl}-2$ was detected. Western blot analysis demonstrated that once MDA-MB-231 cells had been stably transfected with TGM2-shRNA for $96 \mathrm{~h}$, the expression levels of TG2, vimentin and Bcl-2 were significantly downregulated and the expression level of E-cadherin was significantly upregulated, compared with the levels in the NC group ( $\mathrm{P}<0.05$; Fig. 2).

Proliferation of breast cancer cells. The transfected MDA-MB-231 cells were cultured in the presence or absence of TXT for 24,48 or $72 \mathrm{~h}$, and the results showed that the proliferation of the breast cancer cells was strongly inhibited in the RNAi + TXT group, with the difference between the RNAi + TXT group and the other groups being statistically significant at $72 \mathrm{~h}(\mathrm{P}<0.05)$. In addition, the inhibitory level increased in a time-dependent manner (Fig. 3).

Apoptosis rate of MDA-MB-231 cells. Following treatment with TXT for $48 \mathrm{~h}$, the apoptosis of MDA-MB-231 cells was significantly promoted in the RNAi + TXT group $(27.93 \pm 1.1 \%)$ compared with the TXT group $(12.39 \pm 0.67 \%)(\mathrm{P}<0.05)$. RNAi $(6.82 \pm 0.12 \%)$ and TXT alone also promoted the apoptosis of breast cancer cells, however, the effect of RNAi + TXT was the strongest (Fig. 4). Downregulation of TG2 improved the anticancer effect of TXT.

Knockdown of TG2 with shRNA promoted TXT-induced cell death. The combination therapy of TGM2-shRNA with TXT was observed to be more effective than treatment with TXT or RNAi alone or no treatment. This indicates that there are synergistic effects between TGM2-shRNA and TXT, and that the delivery of TGM2-shRNA augmented the TXT-induced apoptosis.

Anticancer effect of combined treatment with TGM2-shRNA and TXT on MDA-MB-231 xenografts

Expression of TG2 within tumors was detected. Immunohistochemical detection of TG2 expression was performed in tissue samples from the xenograft tumors. TG2 was strongly expressed in xenograft tumors from the TXT and NC groups while it was significantly downregulated within tumors from the RNAi and RNAi + TXT groups, which demonstrated that TGM2-shRNA effectively downregulated the expression of TG2 in vivo $(\mathrm{P}<0.05$; Fig. 5$)$.

Average tumor volume. Tumor volume is used to assess the response to antitumor therapy in animal studies. The antitumor effects of the combined treatment with shRNA and TXT were analyzed in a nude mouse model in the present study.

Tumor volume measurements indicated that tumor xenograft growth was suppressed by TGM2-shRNA and TXT. In the RNAi + TXT group the tumor volume remained low, whereas tumors of the TXT group increased in volume. The administration of TXT alone inhibited tumor growth by $28 \%$ and the administration of TGM2-shRNA alone inhibited tumor growth by $\sim 23 \%$ compared with that in the control group, while the combination of TGM2-shRNA and TXT exhibited stronger antitumor effects than either treatment alone, suppressing tumor growth by $\sim 74 \%$ (Fig. 6).

In the RNAi + TXT group, the strongest antitumor effect was observed. The data showed that the combination of TGM2-shRNA and TXT significantly enhanced antitumor 
activity in MDA-MB-231 xenografts, compared with TXT monotherapy, consistent with the results of the in vitro study. TGM2-shRNA effectively modulated the chemosensitivity of breast cancer to TXT.

\section{Discussion}

EMT is characterized by the acquisition of a fibroblast-like cell morphology, the dissolution of tight junctions, cell scattering, the loss of epithelial markers including E-cadherin, and the gain of mesenchymal markers such as vimentin $(8,9)$. MET is the reverse process of EMT, and is a process by which motile mesenchymal cells are converted to polarized epithelial cells. Mesenchymal cells have the ability, which true epithelia do not, to invade and migrate as individual cells through the extracellular matrix constructed by epithelial sheets and by mesenchymal cells themselves (10). As shown in Fig. 1, cells transfected with TGM2-shRNA were rounded with cobblestone epithelial morphology, and organized in tight structures that adopted features of epithelial cells. These characteristics induce adhesion, restrict motility, promote intercellular communication and are frequently identified as well-differentiated (11). Silencing of TGM2 with shRNA changed the expression of EMT markers, such as E-cadherin and vimentin.

The data presented in the present study suggest that the changes observed in TG2-expressing cells were associated with a mesenchymal phenotype. Downregulation of TG2 induces changes associated with EMT, for example, in morphology and EMT-relevant markers, which control chemoresistance (2).

When MDA-MB-231 cells were stably transfected with TGM2-shRNA, the expression of Bcl-2 was significantly downregulated. Bcl-2 is generally regarded as an important anti-apoptotic protein and thus falls into the category of an oncogene. Upregulation of Bcl-2 is common in breast carcinoma (12). TXT-induced apoptosis is linked to the inactivation of Bcl-2 (13). Bcl-2 confers resistance to apoptosis, and prevents TXT-induced apoptosis, thereby reducing the effectiveness of chemotherapy $(14,15)$. The level of TG2 is associated with the expression of Bcl-2 (16): Silencing TGM2 downregulates the level of Bcl-2 (17). The compound rotterlin regulates downstream proteins, including $\mathrm{Bcl}-2$ and nuclear factor $-\kappa \mathrm{B}$, through the TG2/protein kinase $\mathrm{C} \delta$ axis (18). Thus, silencing TGM2 may promote apoptosis and increase the chemosensitivity of MDA-MB-231 cells to TXT by downregulating Bcl-2.

The MTT proliferation assay is broadly used to study the induction and inhibition of cell proliferation, and drug resistance in an in vitro model (19). The transfected MDA-MB-231 cells were treated with TXT and the proliferation inhibition rate of the cells was measured by MTT assay. In the RNAi + TXT group, the proliferation of MDA-MB-231 cells was significantly inhibited compared with that in the control group $(\mathrm{P}<0.05)$, and the level of inhibition increased in a time-dependent manner. Thus, the silencing of TGM2 in breast cancer was found to be associated with decreased tumor cell growth, and improved the anticancer effect of the chemotherapy drug TXT.

The apoptosis of MDA-MB-231 cells was significantly promoted in the RNAi + TXT group compared with the control group. These results suggest that the knockdown of TGM2 in breast cancer is associated with an increased antitumor effect of chemotherapy. Downregulation of TG2 expression promoted TXT-induced cell death. The combination therapy of TGM2-shRNA with TXT was more effective than TXT monotherapy. Hwang et al (5) reported that the combination of TG2 siRNA with TXT in ovarian cancer had a greater efficacy that control siRNA with TXT.

In the present study, treatment with TGM2-shRNA upregulated E-cadherin expression, and downregulated vimentin and Bcl-2 expression in MDA-MB-231 cells. In addition, it significantly increased the TXT-induced inhibition of proliferation and the apoptosis of TNBC cells in vitro. The tumor inhibition rate of the TXT group reached $\sim 38 \%$, compared with that of the combined treatment group. The percentage of tumor inhibition was calculated according to the formula $[1-(\mathrm{R} / \mathrm{N})] \times 100$, where $\mathrm{R}$ and $\mathrm{N}$ represent the mean tumor volumes of the RNAi and NC groups, respectively. Compared with treatment with TXT or RNAi alone, tumor progression was delayed in the RNAi + TXT group. The combination of TGM2-shRNA with TXT demonstrated significant antitumor activity in vivo. These results indicate that the TXT-sensitizing effect of TGM2-knockdown on mammary cancer cells in vitro also took place in vivo. Combination treatment with TGM2-shRNA and TXT appears to be effective against TNBC since TGM2 silencing was found to be effective for the inhibition of cancer cell proliferation in two models of TXT-resistant TNBC.

Numerous lines of evidence supported the role of TG2 overexpression in the pathogenesis and poor clinical outcome of breast cancer. Assi et al (20) reported that patients with stromal TG2 accumulation had significantly decreased disease-free survival (DFS; mean DFS, 110 months) compared with patients with low TG2 expression (mean DFS, 130 months). On the basis of these observations, it may be hypothesized that high TG2 expression levels correlate with poor clinical outcome in patients with breast cancer, which leads to a decreased sensitivity to TXT chemotherapy. Downregulation of TG2 induces changes associated with MET, promotes apoptosis and increases the chemosensitivity of breast cancer.

In conclusion, the current data indicate the important roles of TG2 in the promotion of EMT and drug resistance. Downregulation of TG2 appears to reverse EMT and increase the chemosensitivity of breast cancer to TXT. However, clinical trials are required to clarify whether TG2 expression may be helpful in predicting the treatment efficacy of TXT in patients with breast cancer.

\section{Acknowledgements}

The present study was supported by the Innovation Project of Guangxi Graduate Education of China in 2014 (no. YCBZ2014030).

\section{References}

1. Sarkar FH, Li Y, Wang Z and Kong D: Pancreatic cancer stem cells and EMT in drug resistance and metastasis. Minerva Chir 64: 489-500, 2009

2. Huang RY, Wong MK, Tan TZ, Kuay KT, Ng AH, Chung VY, Chu YS, Matsumura N, Lai HC, Lee YF, et al: An EMT spectrum defines an anoikis-resistant and spheroidogenic intermediate mesenchymal state that is sensitive to e-cadherin restoration by a src-kinase inhibitor, saracatinib (AZD0530). Cell Death Dis 4: e915, 2013. 
3. Yakubov B, Chen L, Belkin AM,Zhang S, Chelladurai B, Zhang ZY and Matei D: Small molecule inhibitors target the tissue transglutaminase and fibronectin interaction. PLoS One 9: e89285, 2014.

4. Kumar A, Xu J, Brady S, Gao H, Yu D, Reuben J and Mehta K Tissue transglutaminase promotes drug resistance and invasion by inducing mesenchymal transition in mammary epithelial cells. PLoS One 5: e13390, 2010.

5. Hwang JY, Mangala LS, Fok JY, Lin YG, Merritt WM, Spannuth WA, Nick AM, Fiterman DJ, Vivas-Mejia PE, Deavers MT, et al: Clinical and biological significance of tissue transglutaminase in ovarian carcinoma. Cancer Res 68 : 5849-5858, 2008.

6. Han HD, Mora EM, Roh JW, Nishimura M, Lee SJ, Stone RL, Bar-Eli M, Lopez-Berestein G and Sood AK: Chitosan hydrogel for localized gene silencing. Cancer Biol Ther 11: 839-845, 2011

7. Shao M, Cao L, Shen C, Satpathy M, Chelladurai B, Bigsby RM, Nakshatri $\mathrm{H}$ and Matei D: Epithelial-to-mesenchymal transition and ovarian tumor progression induced by tissue transglutaminase. Cancer Res 69: 9192-9201, 2009.

8. Nelson CM, Khauv D, Bissell MJ and Radisky DC: Change in cell shape is required for matrix metalloproteinase-induced epithelial-mesenchymal transition of mammary epithelial cells J Cell Biochem 105: 25-33, 2008.

9. Xie L, Law BK, Chytil AM, Brown KA, Aakre ME and Moses HL: Activation of the Erk pathway is required for TGF-beta1-induced EMT in vitro. Neoplasia 6: 603-610, 2004.

10. Yang $J$ and Weinberg RA: Epithelial-mesenchymal transition: At the crossroads of development and tumor metastasis. Dev Cell 14: 818-829, 2008.

11. Christiansen JJ and Rajasekaran AK: Reassessing epithelial to mesenchymal transition as a prerequisite for carcinoma invasion and metastasis. Cancer Res 66: 8319-8326, 2006.
12. Oakes SR, Vaillant F, Lim E, Lee L, Breslin K, Feleppa F, Deb S, Ritchie ME, Takano E, Ward T, et al: Sensitization of BCL-2-expressing breast tumors to chemotherapy by the BH3 mimetic ABT-737. Proc Natl Acad Sci USA 109: 2766-2771, 2012.

13. Nehmé A, Varadarajan P, Sellakumar G, Gerhold M, Niedner H, Zhang Q, Lin X and Christen RD: Modulation of docetaxel-induced apoptosis and cell cycle arrest by all-trans retinoic acid in prostate cancer cells. Br J Cancer 84: 1571-1576, 2001.

14. Emi M, Kim R, Tanabe K, Uchida Y and Toge T: Targeted therapy against Bcl-2-related proteins in breast cancer cells. Breast Cancer Res 7: R940-R952, 2005.

15. Mhaidat NM, Wang Y, Kiejda KA, Zhang XD and Hersey P: Docetaxel-induced apoptosis in melanoma cells is dependent on activation of caspase-2. Mol Cancer Ther 6: 752-761, 2007.

16. Cho SY, Jeong EM, Lee JH, Kim HJ, Lim J, Kim CW, Shin DM, Jeon JH, Choi K and Kim IG: Doxorubicin induces the persistent activation of intracellular transglutaminase 2 that protects from cell death. Mol Cells 33: 235-241, 2012.

17. Kim SJ, Kim KH, Ahn ER, Yoo BC and Kim SY: Depletion of cathepsin $\mathrm{D}$ by transglutaminase 2 through protein cross-linking promotes cell survival. Amino Acids 44: 73-80, 2013.

18. Maioli E, Torricelli $\mathrm{C}$ and Valacchi G: Rottlerin and cancer: Novel evidence and mechanisms. ScientificWorldJournal 2012: $350826,2012$.

19. van Meerloo J, Kaspers GJ and Cloos J: Cell sensitivity assays: The MTT assay. Methods Mol Biol 731: 237-245, 2011.

20. Assi J, Srivastava G, Matta A, Chang MC, Walfish PG and Ralhan R: Transglutaminase 2 overexpression in tumor stroma identifies invasive ductal carcinomas of breast at high risk of recurrence. PLoS One 8: e74437, 2013. 